\title{
Mechanism for synchronized motion between two humans in mutual tapping experiments: Transition from alternative mode to synchronization mode
}

\author{
Yoshikatsu Hayashi ${ }^{1, *}$ and Toshiyuki Kondo ${ }^{2}$ \\ ${ }^{1}$ Cybernetics Group, School of Systems Engineering, University of Reading, P. O. Box 225, Whiteknights, Reading RG6 6AY, United Kingdom \\ ${ }^{2}$ Department of Computer, Information and Communication Sciences, Tokyo University of Agriculture and Technology, \\ 2-24-16, Nakamachi, Koganei-shi, Tokyo 184-8588, Japan
}

(Received 14 February 2013; revised manuscript received 2 July 2013; published 26 August 2013)

\begin{abstract}
We performed mutual tapping experiments between two humans to investigate the conditions required for synchronized motion. A transition from an alternative mode to a synchronization mode was discovered under the same conditions as when a subject changed from a reactive mode to an anticipation mode in single tapping experiments. The experimental results suggest that the cycle time for each tapping motion is tuned by a proportional control that is based on synchronization errors and cycle time errors. As the tapping frequency increases, a mathematical model based on feedback control in the sensory-motor closed loop predicts a discrete transition of the mode as the gain factors of the proportional control decrease. The conditions for synchronization are shown as a consequence of the coupled dynamics based on the next feedback loop in the sensory-motor system.
\end{abstract}

DOI: 10.1103/PhysRevE.88.022715

PACS number(s): 87.19.-j, 05.45.-a, 64.60.De

\section{INTRODUCTION}

Rhythmic behavior in dance and music is an interesting topic from the perspective of synchronization in sensory-motor systems. Humans can simultaneously respond to rhythmic environmental stimuli. However, if these rhythmic stimuli are replaced by motions of other persons, we need a way to achieve synchronized motion and generate a common rhythm. When a generated rhythmic component is shared among members in a group, one can dance or play music in synchronization with other members. In sensory-motor systems, an inevitable time delay is required for processing. Hence, some kind of prediction mechanism is necessary to generate an appropriate behavior for changing environments, or other members of society.

One of the simplest approaches to study sensory-motor synchronization in a laboratory involves finger tapping to a sequence of auditory stimuli. Early investigators noted that taps tended to precede sequence tones by a few tens of milliseconds, rather than being distributed symmetrically around the tone onsets [1-4]. Engström et al. carefully studied the dependency of synchronization errors on tapping frequency and discovered a bimodal peak in the distribution function of the synchronization errors [5]. One peak showed the precedence of the tapping by a few tens of milliseconds. He concluded that the bimodal peak was a reflection of the transition from a reactive to an anticipation mode.

In previous studies of target tracking experiments, we aimed to unveil a mechanism of the transition in the visual-motor system from the feedback corrective motion to proactive control $[6,7]$. We concluded that a generated rhythmic component in the hand motion that was associated with an intermittent error correction gave rise to a proactivity of the hand motion in response to the environmental stimuli [7].

Furthermore, anticipation of chaotic synchronization between a target programmed to move chaotically and a response system with a memory term has been theoretically discussed [8], and its experimental verification was undertaken in a

\footnotetext{
*http://bel.reading.ac.uk/
}

delayed visual tracking system [9]. A tracking experiment for a Rössler chaos of roughly $0.2 \mathrm{~Hz}$ on average with $400 \mathrm{~ms}$ delay to update the visual information revealed anticipatory motion of the subject to compensate the programmed time delay of $400 \mathrm{~ms}$.

On the other hand, mutual interactions have been much less studied. Interpersonal synchronization has recently been studied in the field of walking [10,11]. These authors studied frequency locking, relative phase angle, and the effect of the length difference of the legs on the synchronization. To understand mutual synchronization progress in neuronal research such as investigation of mirror neurons [12] and the role of inhibitory interaction between neurons with time delay will be helpful [13]. However, it might be helpful to understand some common features of mutual interaction by a simpler but better understood experimental setting, such as mutual tracking [14] and mutual tapping.

We need to investigate the principle of mutual behavior that is expected by a subject A when rhythmic flashes of light or sound are replaced by the onset of tapping by another subject B who also tries to tap simultaneously with subject A. The constant proactive behavior or negative asynchrony discovered in mechanical tapping would not be expected by the subject because a mutual tapping experiment is itself symmetric.

In this study, we investigated motional communication using mutual tapping experiments in which the periodic stimuli used in the previous studies were replaced by the tapping of another human being. This study aims to verify the mechanism that causes adjustments of appropriate control parameters and results in the characteristic transition from the alternative mode to the synchronization mode. Therefore, we eventually investigate a mechanism in which real-time synchronization is realized.

\section{EXPERIMENT}

\section{A. Experimental procedure}

The subjects were instructed to coordinate the tapping onsets of their preferred index finger in response to flashes 


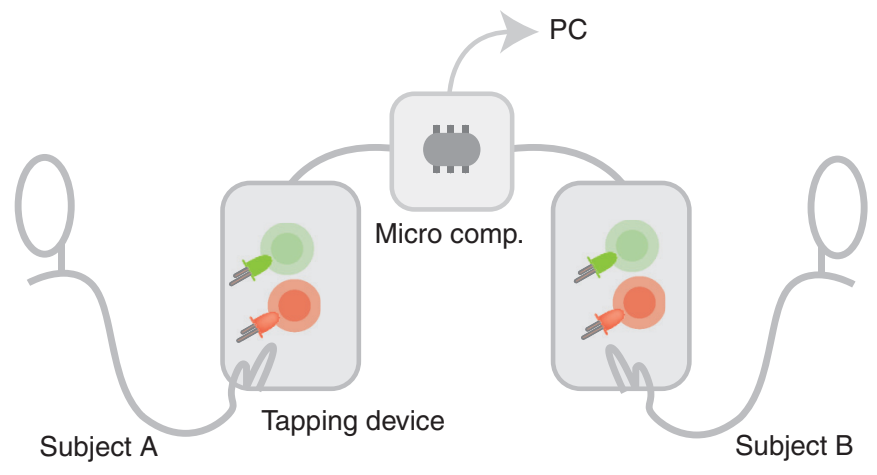

FIG. 1. (Color online) Schematic picture of the experimental system in the mutual tapping experiment; two tapping devices were connected through a microcomputer. The microcomputer was connected to a PC using serial transmission to transmit the tapping data.

of light. In the mutual tapping experiments we constructed a cross-feedback system between two tapping devices in which a touch sensor was placed to detect the onset of tapping (Fig. 1).

By connecting two tapping devices through a microcomputer (ATMEGA 168, Atmel Corp.), a tapping signal of subject A was transmitted to subject B. Two tapping devices transmitted signals to the microcomputer by a transistortransistor-logic (TTL) circuit, and vice versa, triggering the LED to flash. The onset of tapping was recorded every $5 \mathrm{~ms}$ and was transmitted to the other PC through serial communication. Software (LABVIEW, National Instruments) was used to analyze the tapping data in real-time processing.

Two subjects were instructed to coordinate the tapping onsets of their index fingers so that each tapping onset occurred simultaneously with light flashes. To regulate the mutual tapping frequency, the flash of a green LED light at a certain frequency was presented five times prior to mutual tapping; this is referred to as mechanical tapping. We performed two types of mutual tapping experiments depending on the aim of the analysis:

(1) Consecutive analysis: the initial frequency of light was set in the range of $0.3-1.6 \mathrm{~Hz}$ with small increments of $0.1 \mathrm{~Hz}$.

(2) Statistical analysis: the initial frequency of light was set to be $0.5,0.7$, and $1.0 \mathrm{~Hz}$.

The tapping tasks were performed in an ascending series of the programmed frequency.

After mechanical tapping, the flashing green light was switched to red light (Fig. 1). The flash of the red light was synchronized with the tapping onset of the partner in the cross-feedback system. Thus, subjects were notified when the mutual tapping started by the changing color of the LED light. The mutual tapping was set to include 33 taps of the index finger. Subjects were seated comfortably in a chair in front of the computer. The two subjects sat beside each other at a fixed distance (about $1 \mathrm{~m}$ ) at which they felt comfortable. The height of the chair was adjusted such that the upper limbs rested horizontally on a padded support.

After the subjects were in the correct posture, instructions were issued. Each subject was given a description of the current experimental conditions (tapping frequency and trial number) and the instructions listed below:

(1) Maintain the initial frequency as accurately as possible while performing mutual tapping.
(2) As much as possible, simultaneously tap in response to the flashes of light.

The detailed experimental procedure is given as follows. We employed two types of experimental conditions. In the case of consecutive tapping experiments:

(1) One trial consisted of 5 initial mechanical taps and 33 mutual taps.

(2) After each trial, the subjects rested for about 1 or $2 \mathrm{~min}$, or upon request.

(3) The initial target frequency was progressively increased from 0.3 to $1.6 \mathrm{~Hz}$ in steps of $0.1 \mathrm{~Hz}$.

(4) The subjects performed a total of 12 trials, which were referred as one set.

In the case of tapping experiments for statistical analysis:

(1) One trial consisted of 5 initial mechanical taps and 33 mutual taps.

(2) After each trial, subjects rested for about 1 or $2 \mathrm{~min}$, or upon request.

(3) The initial target frequency was progressively increased from 0.5 to $0.7 \mathrm{~Hz}$ to $1.0 \mathrm{~Hz}$.

(4) The subjects performed three trials for each frequency (nine trials in total), which were referred as one set.

\section{B. Subject information}

In all experimental conditions, the subjects were well trained in mechanical tapping experiments where an external rhythmic signal is given. The subjects consisted of five males and one female in their twenties, from whom seven pairs were arranged. Four of them were selected to make six pairs (full combination) and two of them were selected to make one pair. In the last pair, one of the members was left handed. The index finger used for tapping was on the dominant hand. The institutional ethics committee at the Tokyo University of Agriculture and Technology approved the procedure of the experiments and the subjects gave informed consent before participation. In order to achieve relatively stable mutual tapping, i.e., for the cycle of flexion to relax to a certain frequency, several trials were carried out for each pair at each frequency. As a result, the tapping motion did not become significantly faster or slower over the mutual tapping period.

\section{Analysis method}

The first three sets of tapping data during the mutual tapping period were omitted and the data of 30 tapping trials were used for the following analysis. Characteristic features of the tapping motion were represented by synchronization errors and cycle time errors between two persons (Fig. 2). The time difference between the tapping onsets of subjects A and B was recorded for each tap as the synchronization error. The synchronization errors $\left(S_{E}\right)$ between the tapping motions of each subject were given by

$S_{E, A}(n)=T_{B}(n)-T_{A}(n), \quad S_{E, B}(n)=T_{A}(n)-T_{B}(n)$,

where $S_{E, A}(n)$ represents the synchronization error for subject A and $S_{E, B}(n)$ represents that for subject B. $T_{A}(n)$ and $T_{B}(n)$ denote the timing of the $n$th tapping onsets of subjects $\mathrm{A}$ and $\mathrm{B}$, respectively. 


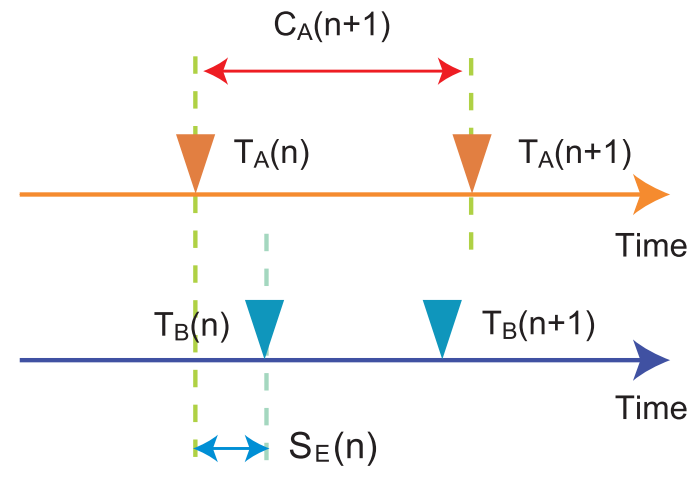

FIG. 2. (Color online) Analysis of mutual tapping experiment; the cycle time $C_{A}(n+1)$ and the synchronization error $S_{E}(n)$ were calculated to characterize the tapping behavior.

The cycle time of tapping was obtained by subtracting the timing of the $n$th taps from the timing of the $(n+1)$ th taps:

$$
\begin{aligned}
& C_{A}(n+1)=T_{A}(n+1)-T_{A}(n), \\
& C_{B}(n+1)=T_{B}(n+1)-T_{B}(n) .
\end{aligned}
$$

The cycle time errors $\left(C_{E}\right)$ of the $n$th taps between the pair were calculated for each subject as follows:

$$
\begin{aligned}
& C_{E, A}(n)=C_{B}(n)-C_{A}(n), \\
& C_{E, B}(n)=C_{A}(n)-C_{B}(n) .
\end{aligned}
$$

\section{RESULTS AND DISCUSSION}

\section{A. Emergence of synchronization}

We first focus on the time development for the cycle time in a pair. The typical time development for the cycle time is shown in Fig. 3(a) when the initial frequency is $0.5 \mathrm{~Hz}$. The pair of subjects alternately tunes the cycle time from one tap to another. One subject shows higher amplitude than the other. The average cycle times of subjects A and B are 2047 and $2041 \mathrm{~ms}$, respectively. The pair can maintain the initial frequency well during the mutual tapping period. The $S_{E}$ between the pair oscillates in turn between positive and negative values [Fig. 3(b)].

When the mutual tapping frequency increases up to $1.0 \mathrm{~Hz}$ (Fig. 4), the amplitude of the alternative oscillation decreases. Figure 4(b) shows the time development of $S_{E}$. $S_{E}$ has a periodic behavior as a function of time; however, the fluctuation of $S_{E}$ is more suppressed than that at the lower frequency.

Figure 5 shows the standard deviation of $S_{E}$ obtained from one trial as a function of initial tapping frequency. The data were collected from seven pairs. As the initial frequency increases, the $S_{E}$ between two subjects rapidly decreases. From the distribution of the standard deviation, two peaks were found (Fig. 6). A threshold of $50 \mathrm{~ms}$ can be introduced to distinguish between two tapping modes, nonsynchronization and synchronization modes $(p<0.05$ in an independent sample $t$ test). We considered that the synchronization in mutual tapping occurred when the standard deviation became less than $50 \mathrm{~ms}$.

The transition region of the two modes from the alternative mode to the synchronization mode was found to range from 0.5 to $1.0 \mathrm{~Hz}$, depending on the pair (Fig. 5). This transition
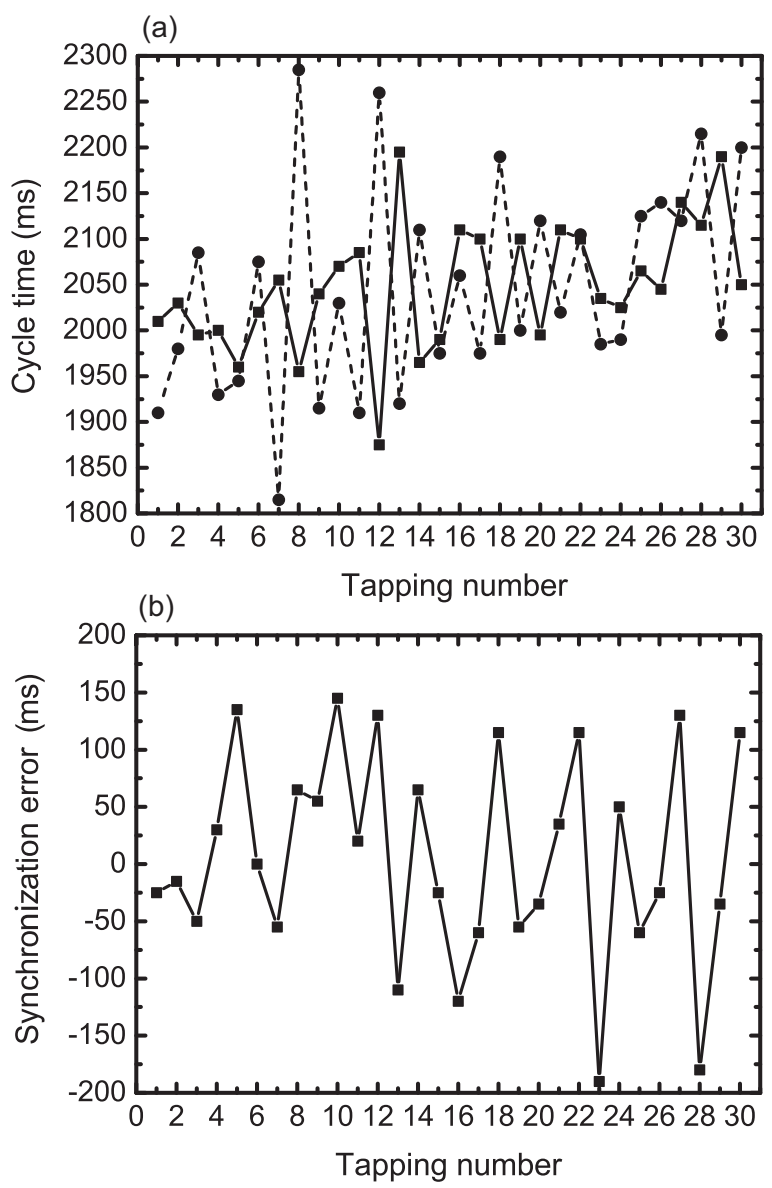

FIG. 3. Typical time development for tapping behavior when the initial frequency was $0.5 \mathrm{~Hz}$. (a) Cycle time of each subject; the solid line represents the cycle time of subject A and the dashed line represents that of subject B. (b) Synchronization error of subject A.

region corresponds to the transition region in single tapping experiments, as reported by Engström et al. [5].

In mechanical tapping experiments, when rhythmic stimuli are provided, responses of humans are caused by each environmental stimulus in a reactive mode, i.e., the subjects tap immediately after detecting the external signal. On the other hand, in mutual tapping experiments, both subjects in the pair cannot simultaneously wait for the other to tap, and so they have an urge to tap spontaneously. The subject should determine the moment of the next tap with respect to the time difference between the external stimulus and self-tap. Therefore, some kind of algorithm is required to determine the moment of the next tap.

\section{B. Proportional control based on $S_{E}$}

To investigate the mechanism of transition based on error correction, or the control law of tapping, we first investigate whether the cycle time differences should linearly depend on $S_{E}$ as expressed by the following equations:

$$
\begin{aligned}
& C_{A}(n+1)-C_{A}(n)=k_{S, A} S_{E, A}(n), \\
& C_{B}(n+1)-C_{B}(n)=k_{S, B} S_{E, B}(n),
\end{aligned}
$$




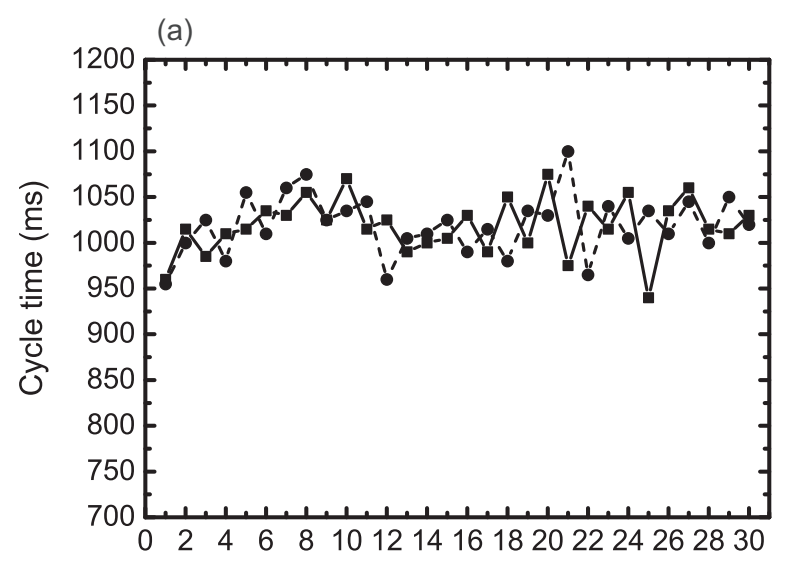

(b)

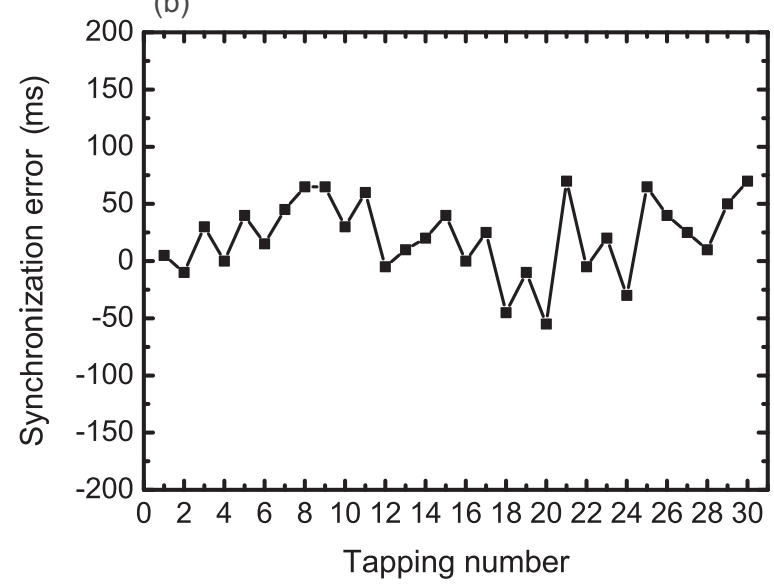

FIG. 4. Typical time development for tapping behavior when the initial frequency was $1.0 \mathrm{~Hz}$. (a) Cycle time of each subject; the solid line represents the cycle time of subject $A$ and the dashed line represents that of subject B. (b) Synchronization error of subject A.

where $k_{S, A}$ and $k_{S, B}$ are gain factors in a proportional control for subjects $\mathrm{A}$ and $\mathrm{B}$, respectively.

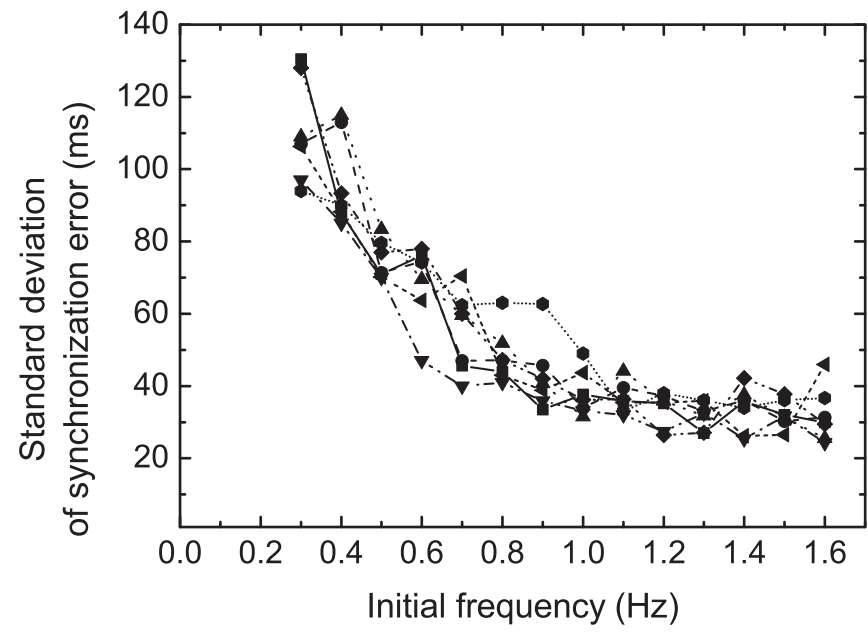

FIG. 5. Standard deviation of synchronization errors as a function of initial tapping frequency. Each data point was calculated from one trial. A symbol represents a particular pair. The data were collected from seven pairs.

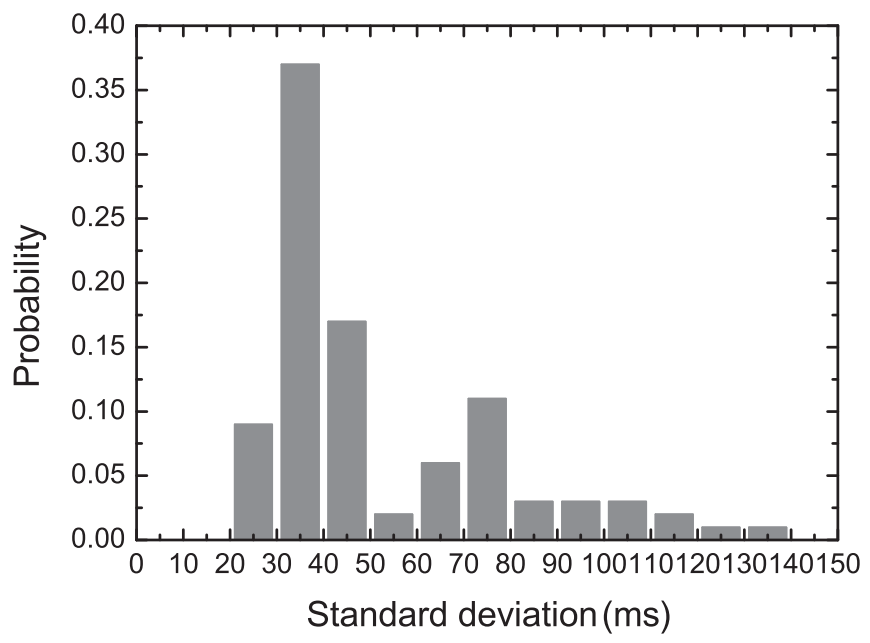

FIG. 6. Distribution of the standard deviation of synchronization errors. A threshold of $50 \mathrm{~ms}$ can be introduced to distinguish between the two distributions ( $p<0.05$ in an independent sample $t$ test). The data were collected from seven pairs.

To experimentally verify the linear dependence described by Eq. (4), the cycle time differences were plotted as a function of $S_{E}$ and a linear fitting was performed to calculate $k_{C, A}, k_{C, B}$, and the coefficient of determination $R^{2}$ at each frequency. Three frequencies $(0.5,0.7$, and $1.0 \mathrm{~Hz})$ were selected and data from three trials at each frequency were accumulated to statistically calculate $k_{C, A}, k_{C, B}$, and $R^{2}$, as shown in Fig. 7. At the frequency of $0.5 \mathrm{~Hz}$, the $R^{2}$ was greater than 0.7 and at higher frequencies greater than 0.38 . The other six pairs showed the same trend. Thus, we confirmed that Eq. (4) is a good approximation. Humans tuned the tapping cycle time to be proportional to $S_{E}$.

This behavior can be regarded as a proportional control of the cycle time based on $S_{E}$ and is simply obtained from Eq. (4):

$$
\begin{aligned}
& C_{A}(n+1)=C_{A}(n)+k_{S, A} S_{E, A}(n), \\
& C_{B}(n+1)=C_{B}(n)+k_{S, B} S_{E, B}(n),
\end{aligned}
$$

where $k_{S, A}$ and $k_{S, B}$ can be regarded as gain factors by which the corresponding $S_{E}$ is weighted for corrections. These gain factors represent a subject's performance strategy and can have different values for each subject.

From the results for the seven pairs, we found that the sum of proportionality factors decreased as the initial tapping frequency increased (Fig. 8). Depending on the pace of self-motion, subjects change the proportionality factors in the proportional control. This experimental fact indicates that sensitivity of the time difference between the tactile sensation and the flash of light changes in each individual as a function of the tapping frequency.

More interestingly, one member of the pair has a higher value of gain factor at the initial frequency of $0.5 \mathrm{~Hz}$ (Fig. 9) and another member is more sensitive to $S_{E}$. In the process of proportional control, this can be considered as a division of roles. It would be interesting to observe the dependence of the gain factors on each combination of pairs.

Table I shows the member of each pair that has a higher value of $k_{S}$ at the initial frequency of $0.5 \mathrm{~Hz}$. The gain factor in the control law based on $S_{E}$ is determined by each pair 
(1a)

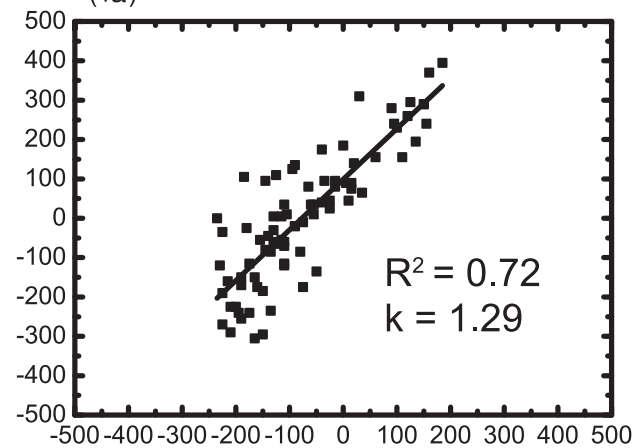

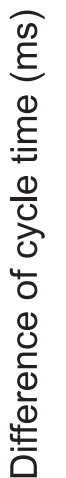

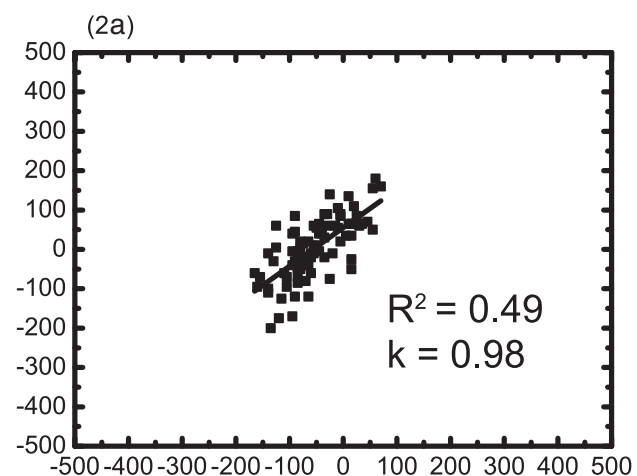

(3a)

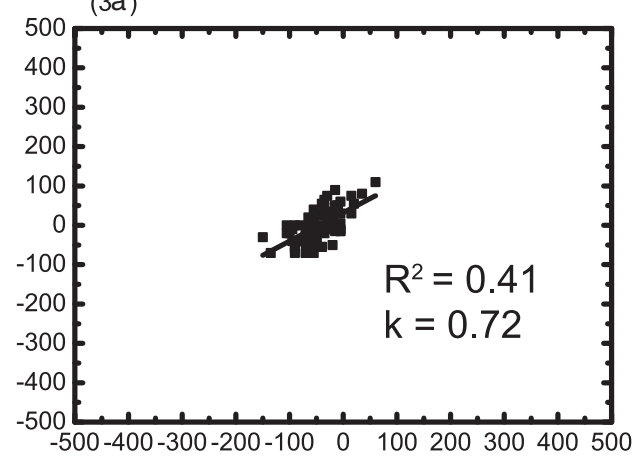

(1b)

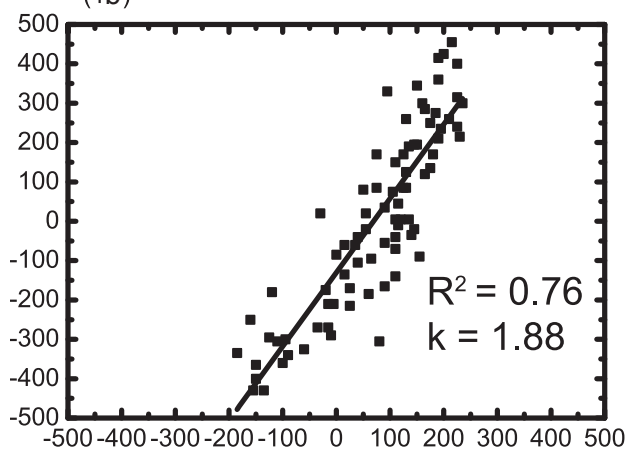

(2b)

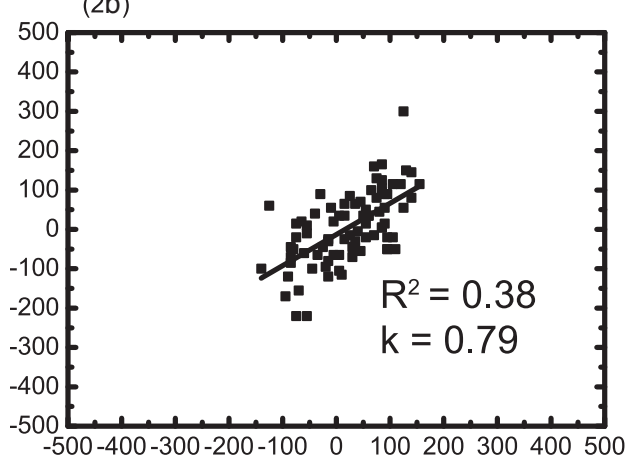

(3b)

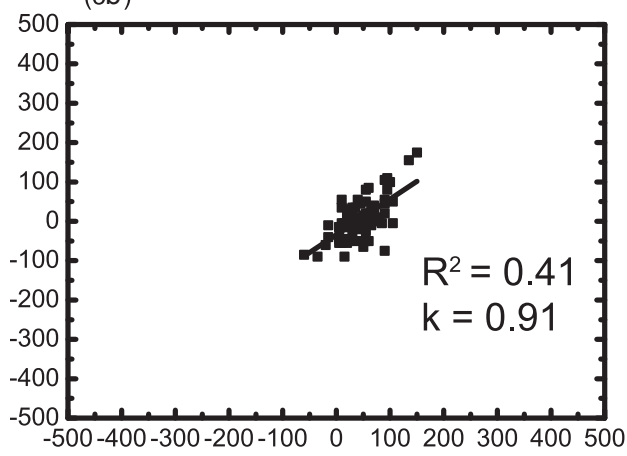

Synchronization error (ms)

FIG. 7. Typical example of the difference in cycle time as a function of $S_{E}$. (1a) Initial frequency $0.5 \mathrm{~Hz}$ for subject A. (1b) Initial frequency $0.5 \mathrm{~Hz}$ for subject B. (2a) Initial frequency $0.7 \mathrm{~Hz}$ for subject A. (2b) Initial frequency $0.7 \mathrm{~Hz}$ for subject B. (3a) Initial frequency $1.0 \mathrm{~Hz}$ for subject A. (3b) Initial frequency $1.0 \mathrm{~Hz}$ for subject B. The data were collected from one pair.

while mutually tapping. A certain subject does not always have a value of $k_{S}$ that is higher than those of the other three partners. We found that the division of roles for gain factors was a characteristic feature determined in the mutual tapping of each pair.

\section{Proportional control based on $\boldsymbol{C}_{\boldsymbol{E}}$}

Next, we check whether the cycle time difference should linearly depend on $C_{E}$ between two subjects, as expressed by the following equations:

$$
\begin{aligned}
& C_{A}(n+1)-C_{A}(n)=k_{C, A} C_{E, A}(n), \\
& C_{B}(n+1)-C_{B}(n)=k_{C, B} C_{E, B}(n),
\end{aligned}
$$

where $k_{C, A}$ and $k_{C, B}$ are the gain factors in the proportional control corresponding to subjects $\mathrm{A}$ and $\mathrm{B}$, respectively. To experimentally verify the linear dependence, the cycle time difference was plotted as a function of $C_{E}$ and a linear fitting was performed to calculate $k_{C, A}, k_{C, B}$, and $R^{2}$. Three frequencies $(0.5,0.7$, and $1.0 \mathrm{~Hz})$ were selected and the data from the three trials in each frequency were accumulated to calculate statistical values.

Figure 10 illustrates a typical example of the difference in the cycle time as a function of $C_{E}$. One can clearly see the linear dependence of the cycle time difference on $C_{E}$. It was found that the lowest value of $R^{2}$ was 0.56 , at the frequency of $1.0 \mathrm{~Hz}$. The other six pairs showed the same trend. From Eq. (6), we can obtain the following equations as a control law:

$$
\begin{aligned}
& C_{A}(n+1)=C_{A}(n)+k_{C, A} C_{E, A}(n), \\
& C_{B}(n+1)=C_{B}(n)+k_{C, B} C_{E, B}(n),
\end{aligned}
$$




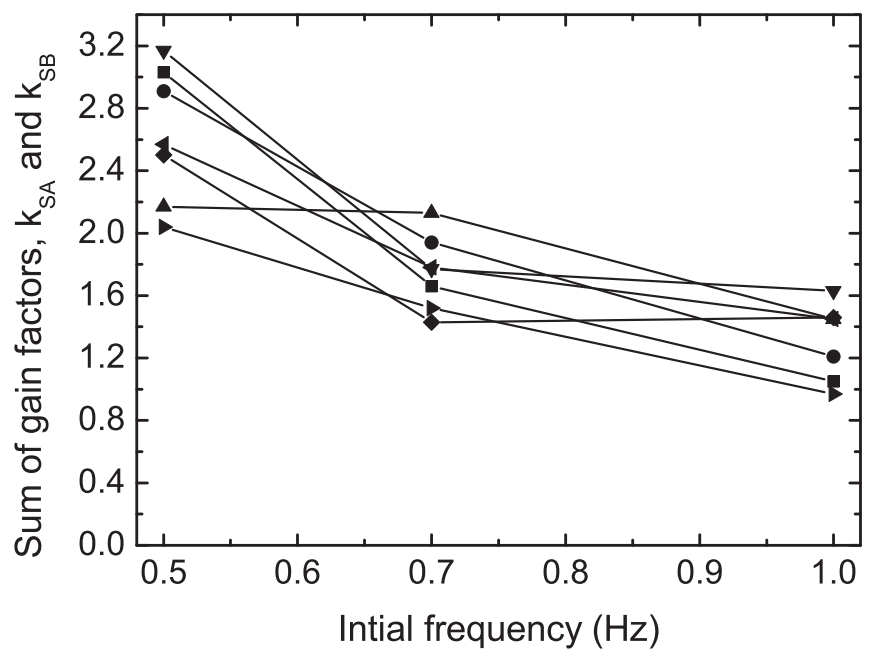

FIG. 8. Sum of gain factors for synchronization errors as a function of the initial frequency. Each symbol represents a particular pair. The data were collected from seven pairs.

where $k_{C, A}$ and $k_{C, B}$ can be regarded as gain factors by which the corresponding $C_{E}$ is weighted for corrections. These gain factors represent a subject's performance strategy and can have different values for each subject.

We found that the sum of the proportionality factors decreased as the initial tapping frequency increased (Fig. 11). At lower frequencies, one member of the pair has a higher value of the proportionality factor than the partner (Fig. 12). Alternatively, one can be considered to be more sensitive to the time difference between the self-motion and the motion of others. This can be considered as a division of roles, which was discussed in the previous section. Table II identifies the member in each pair that has a higher value of $k_{C}$ at the initial frequency of $0.5 \mathrm{~Hz}$. A certain subject does not always have the higher value of $k_{C}$ when compared to the value of the partner, with the exception of subject $\mathrm{C}$. The sensitivity of $C_{E}$ is determined by each pair. As discussed in the section concerning proportional control based on $S_{E}$, we found that

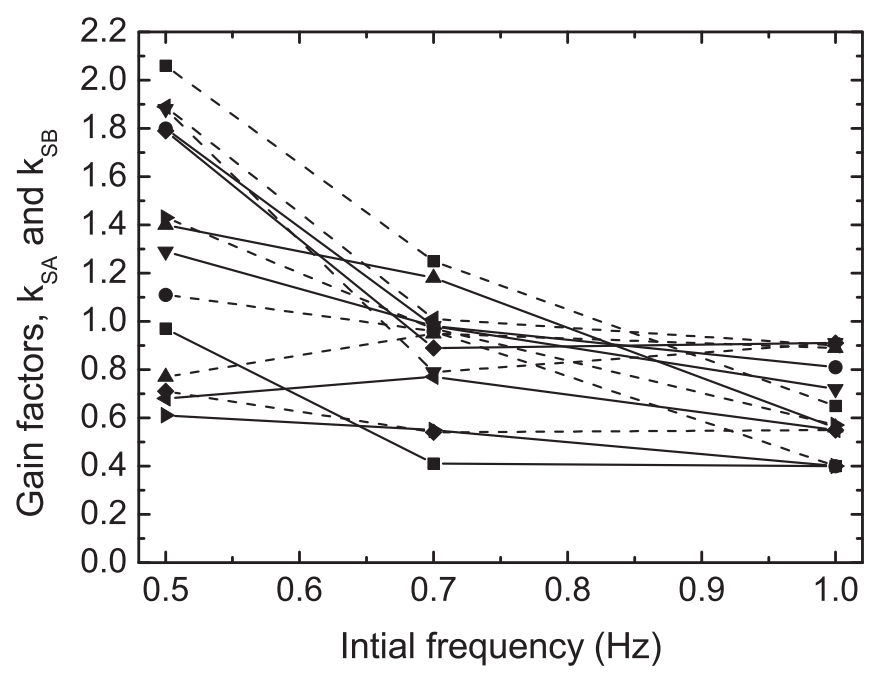

FIG. 9. Gain factors for synchronization errors as a function of the initial frequency. Each symbol represents a particular pair. The data were collected from seven pairs.
TABLE I. Standings of four subjects on gain factors; the capital letter represents the subject identification. " 1 " denotes the case when the subject in the horizontal line has a higher $k_{S}$ value than that of the subject in the perpendicular line. " 0 " denotes the opposite case. The initial frequency was set to $0.5 \mathrm{~Hz}$.

\begin{tabular}{lllll}
\hline \hline & A & B & C & D \\
\hline A & & 0 & 1 & 1 \\
B & 1 & & 0 & 0 \\
C & 0 & 1 & & 1 \\
D & 0 & 1 & 0 & \\
\hline \hline
\end{tabular}

the division of roles was determined by each pair in the process of mutual tapping.

\section{MODELING}

From the experimental results we concluded that the cycle time correction was performed by the proportional control based on $S_{E}$ as described by Eq. (5) and on $C_{E}$ by Eq. (7). In this section we calculate an analytical solution for $S_{E}$ and discuss conditions for its convergence.

\section{A. Proportional control based on $S_{E}$}

First, we derive an analytical solution for $S_{E}$ tuned by the proportional control based on $S_{E}$. Substituting the definitions of $S_{E}$ and cycle time [Eqs. (1) and(2)] into the equation of proportional control [Eq. (5)], we obtain the solution for $S_{E, A}(n)$ :

$$
S_{E, A}(n)= \begin{cases}C_{1} \alpha^{n-1}-C_{2} \beta^{n-1} & (\alpha \neq \beta), \\ C_{1} \alpha^{n-1}-C_{2} n \alpha^{n-1} & (\alpha=\beta),\end{cases}
$$

where $C_{1}$ and $C_{2}$ are numerical constants that consist of the initial values of $S_{E, A}$, and the values of $\alpha$ and $\beta$ are determined as follows:

$$
\begin{aligned}
& \alpha=-\left(\frac{k_{S, A}+k_{S, B}}{2}-1\right)+\sqrt{\left(\frac{k_{S, A}+k_{S, B}}{2}-1\right)^{2}-1,} \\
& \beta=-\left(\frac{k_{S, A}+k_{S, B}}{2}-1\right)-\sqrt{\left(\frac{k_{S, A}+k_{S, B}}{2}-1\right)^{2}-1 .}
\end{aligned}
$$

For $4<k_{S, A}+k_{S, B}$, we obtain $\alpha, \beta \in \mathbf{R}$. In this case, $|\beta|>1$, giving the divergence in Eq. (8). For $k_{S, A}+k_{S, B}=4$, the solution in Eq. (8) is periodic. For $0 \leqslant k_{S, A}+k_{S, B}<4$, we obtain $\alpha, \beta \in \mathbf{C}$. The complex conjugate numbers can be generally written as $\alpha=A e^{i \theta}$ and $\beta=A e^{-i \theta}$, and the complex constants are written as $C_{1}=s_{1}+i t_{1}$ and $C_{2}=s_{2}+i t_{2}$. Thus, Eq. (8) becomes

$$
\begin{aligned}
S_{E}(n)= & C_{1} \alpha^{n}+C_{2} \beta^{n} \\
= & A^{n}\left[\left(s_{1}+s_{2}\right) \cos (n \theta)-\left(t_{1}-t_{2}\right) \sin (n \theta)\right] \\
& +i A^{n}\left[\left(s_{1}-s_{2}\right) \sin (n \theta)+\left(t_{1}+t_{2}\right) \cos (n \theta)\right] .
\end{aligned}
$$

For $n=0, n=1, S_{E}(0) \in \mathbf{R}$, and $S_{E}(1) \in \mathbf{R}$, we obtain $s_{1}-$ $s_{2}=0$ and $t_{1}+t_{2}=0$. Therefore, Eq. (8) becomes

$$
S_{E}(n)=A^{n}\left[\left(s_{1}+s_{2}\right) \cos (n \theta)-\left(t_{1}-t_{2}\right) \sin (n \theta)\right] .
$$



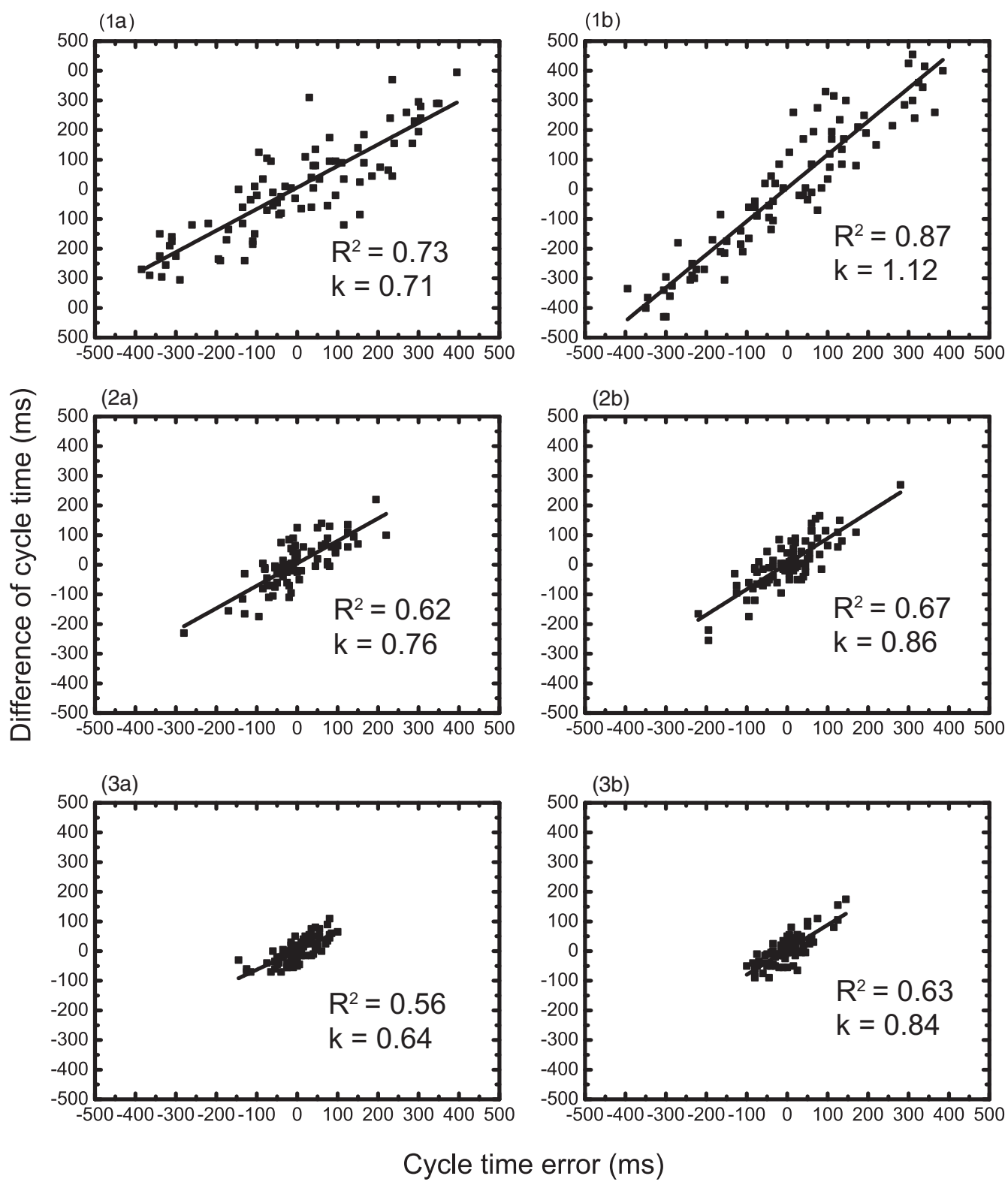

FIG. 10. Typical example of the difference in cycle time as a function of $C_{E}$. (1a) Initial frequency $0.5 \mathrm{~Hz}$ for subject $\mathrm{A}$. (1b) Initial frequency $0.5 \mathrm{~Hz}$ for subject B. (2a) Initial frequency $0.7 \mathrm{~Hz}$ for subject A. (2b) Initial frequency $0.7 \mathrm{~Hz}$ for subject B. (3a) Initial frequency $1.0 \mathrm{~Hz}$ for subject A. (3b) Initial frequency $1.0 \mathrm{~Hz}$ for subject B. The data were collected from one pair.

The product of complex conjugate numbers is given by $\alpha \beta=$ $A^{2}$. From Eq. (9), we immediately find $\alpha \beta=1$. Therefore, $A=1$ is obtained in Eq. (10), which does not lead to the convergence in the periodic behavior.

In summary, we obtain the following conditions for the time development of $S_{E}$.

(1) For the condition of gain factors $0 \leqslant k_{S, A}+k_{S, B} \leqslant 4$, $S_{E}$ has a periodic behavior as a function of the tapping number.

(2) For the condition of gain factors $4<k_{S, A}+k_{S, B}, S_{E}$ diverges as a function of the tapping number.

From the experimental results (Fig. 8), the first condition is satisfied over the examined frequency range. Thus, via proportional control based on $S_{E}$, a periodic behavior of $S_{E}$ should be expected over the entire frequency range.

\section{B. Proportional control based on $C_{E}$}

From the experimental results, we also concluded that the cycle time correction was based on $C_{E}$. From Eq. (7), we can also develop the solution for $S_{E, A}(n)$ :

$$
S_{E, A}(n)=\frac{1}{k_{C, A}+k_{C, B}}\left\{C_{1}-C_{2}\left[1-\left(k_{C, A}+k_{C, B}\right)\right]^{n-1}\right\},
$$

where $C_{1}$ and $C_{2}$ are some numerical constants. Thus, we found the following conditions:

(1) For the condition of gain factors $k_{C, A}+k_{C, B}>2, S_{E}$ diverges as the number of taps increases.

(2) For the condition of gain factors $0<k_{C, A}+k_{C, B}<2$, $S_{E}$ converges to a certain value. 


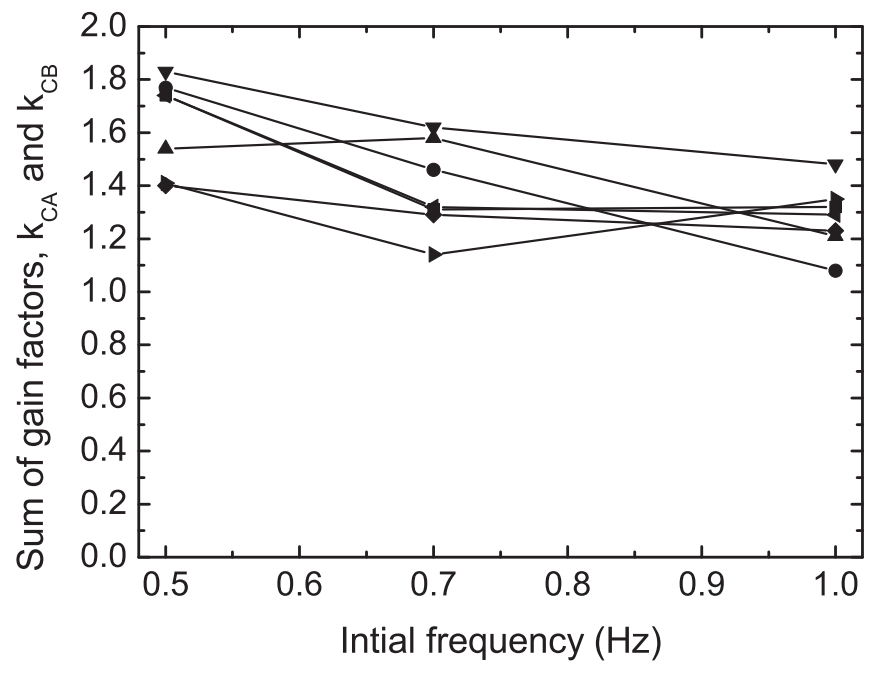

FIG. 11. Sum of gain factors for cycle time errors as a function of initial frequency. Each symbol represents a particular pair. The data were collected from seven pairs.

From the experimental results for the gain factors shown in Fig. 12, we can see that the second condition has been satisfied. Thus, via proportional control based on $C_{E}, S_{E}$ should converge over the entire frequency range examined in the present mutual tapping experiments.

\section{Combination model}

In previous sections, we considered two types of control law based on the $S_{E}$ and $C_{E}$ between the pair. The former control leads to a periodic solution of $S_{E}$ and the latter leads to convergence of $S_{E}$. However, $S_{E}$ and $C_{E}$ are not independent quantities as shown below:

$$
\begin{aligned}
C_{E, A}(n) & =C_{B}(n)-C_{A}(n) \\
& =\left[T_{B}(n)-T_{B}(n-1)\right]-\left[T_{A}(n)-T_{A}(n-1)\right] \\
& =S_{E, A}(n)-S_{E, A}(n-1) .
\end{aligned}
$$

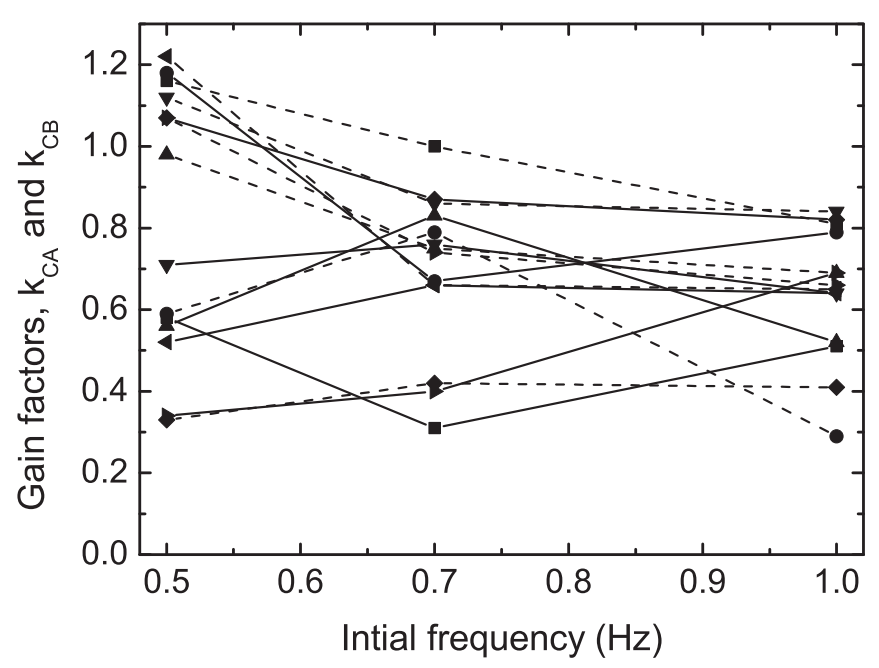

FIG. 12. Gain factors for the cycle time errors as a function of initial frequency. Each symbol represents a particular pair. The data were collected from seven pairs.
TABLE II. Standings of four subjects on gain factors; the capital letter represents the subject identification. " 1 " denotes the case when the subject in the horizontal line has a higher $k_{C}$ value than that of the subject in the perpendicular line. " 0 " denotes the opposite case. The initial frequency was set to $0.5 \mathrm{~Hz}$.

\begin{tabular}{lllll}
\hline \hline & A & B & C & D \\
\hline A & & 0 & 1 & 1 \\
B & 1 & & 0 & 1 \\
C & 0 & 1 & & 1 \\
D & 0 & 0 & 0 & \\
\hline \hline
\end{tabular}

Indeed, $C_{E}$ consists of the differentiation of $S_{E}$. The experimental results also suggested that two types of proportional control should function together in a single trial.

In this section, we turn on these two types of proportional control functioning together. As a first step for the unified control, it appears plausible to consider the case when two proportional control laws function simultaneously. If the proportional control functions are based on $S_{E}$ and $C_{E}$ simultaneously, the following equations can be applied:

$$
\begin{aligned}
& C_{A}(n+1)=C_{A}(n)+k_{A} S_{E, A}(n)-k_{A}^{\prime} S_{E, A}(n-1), \\
& C_{B}(n+1)=C_{B}(n)+k_{B} S_{E, B}(n)-k_{B}^{\prime} S_{E, B}(n-1) .
\end{aligned}
$$

By substituting the definition of the cycle time [Eq. (2)], we obtain an analytical solution for $S_{E}$ :

$$
S_{E, A}(n)= \begin{cases}C_{1} \alpha^{n-1}-C_{2} \beta^{n-1} & (\alpha \neq \beta), \\ C_{1} \alpha^{n-1}-C_{2} n \alpha^{n-1} & (\alpha=\beta),\end{cases}
$$

where $\alpha$ and $\beta$ are respectively given as follows:

$$
\begin{aligned}
& \alpha=\frac{-(K-2)+\sqrt{(K-2)^{2}-4\left(1-K^{\prime}\right)}}{2}, \\
& \beta=\frac{-(K-2)-\sqrt{(K-2)^{2}-4\left(1-K^{\prime}\right)}}{2} .
\end{aligned}
$$

Here, we introduced $K$ to express the sum of $k_{A}$ and $k_{B}$ ( $K=$ $\left.k_{A}+k_{B}\right)$ and $K^{\prime}$ to express the sum of $k_{A}^{\prime}$ and $k_{B}^{\prime}\left(K^{\prime}=\right.$ $k_{A}^{\prime}+k_{B}^{\prime}$ ). While $\alpha \in \mathbf{R}, \beta \in \mathbf{R}$, the absolute value of $|\alpha|$ and $|\beta|$ should be less than unity for convergence $(|\alpha| \leqslant 1,|\beta| \leqslant$ 1). Therefore, the condition of convergence in Eq. (15) was obtained as follows:

$$
K^{\prime} \leqslant-K+4, \quad K^{\prime} \leqslant K, \quad K^{\prime} \geqslant-\frac{K^{2}}{4}+4 .
$$

Furthermore, under the condition $K^{\prime}<-\frac{K^{2}}{4}+4, \alpha$ and $\beta$ become complex conjugate numbers $\alpha \in \mathbf{C}, \beta \in \mathbf{C}$, and the solution is given by

$$
S_{E}(n)=A^{n}\left[\left(s_{1}+s_{2}\right) \cos (n \theta)-\left(t_{1}-t_{2}\right) \sin (n \theta)\right]
$$

with $A=1-K^{\prime}$. Therefore, the periodic solution in Eq. (17) converges for $0<K^{\prime}$. Now the convergence condition is given by

$$
K^{\prime} \leqslant-K+4, \quad K^{\prime} \leqslant K, \quad 0<K^{\prime} .
$$

Figure 13 shows the $K-K^{\prime}$ diagram to represent the conditions for convergence and divergence of $S_{E}$. It is straightforward to see that the $S_{E}$ model corresponds to the condition $K^{\prime}=0$ 


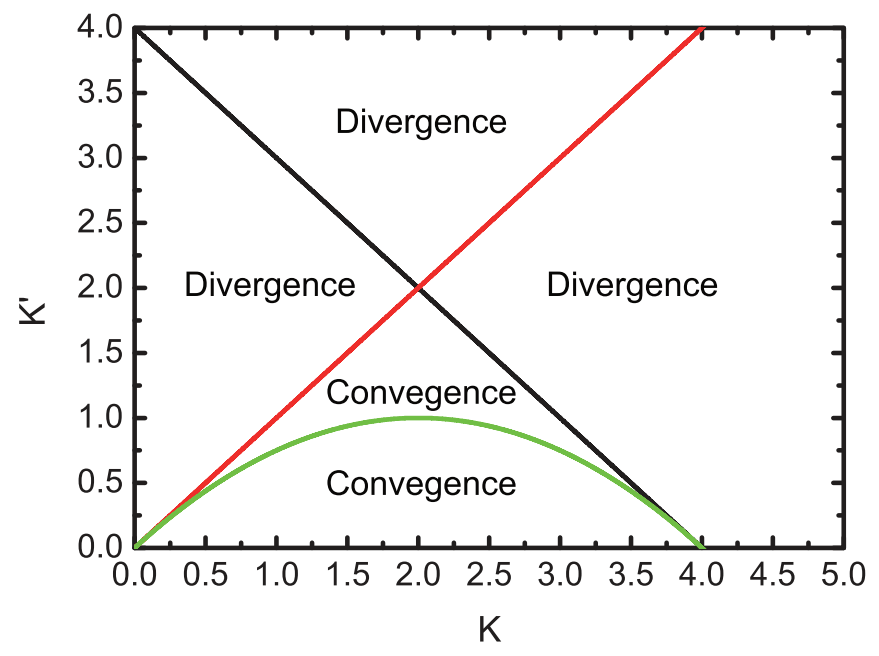

FIG. 13. (Color online) $K-K^{\prime}$ diagram of conditions for convergence and divergence in the combination model as obtained from Eq. (18).

and that the $C_{E}$ model corresponds to the condition $K^{\prime}=K$. Thus, the conditions for convergence of $S_{E}$ are consistent with the results obtained in the previous sections.

As a first attempt, the coefficients in Eq. (13) can be associated with the coefficients in Eq. (5). We regard $k_{A}$ and $k_{B}$ in Eq. (13) as $k_{S, A}$ and $k_{S, B}$ in Eq. (5), respectively. Next, a correspondence between the coefficients of Eqs. (13) and (7) may be considered: $k_{A}^{\prime}$ and $k_{B}^{\prime}$ in Eq. (13) are regarded as $k_{C, A}$ and $k_{C, B}$ in Eq. (7), respectively.

Then, we apply the experimentally obtained numerical values of the gain factors to the equation of the combination model. We can confirm that the sum of $K^{\prime}$ and $K$ is greater than 4.0 for five pairs (Figs. 8 and 11) when the tapping frequency is $0.5 \mathrm{~Hz}$. This experimental result actually indicates the condition of divergence in Eq. (18) and Fig. 13. As the tapping frequency increases, the sum of $K^{\prime}$ and $K$ decreases below 4.0 for five pairs (Figs. 8 and 11). This result indicates the condition of convergence in Eq. (18), crossing a line into the triangle of convergence in Fig. 13. The second condition $\left(K^{\prime} \leqslant K\right)$ is also satisfied in the frequency range examined with the exception of one pair.

It is interesting that the triangle configuration of the convergence region in Fig. 13 guarantees synchronization in the case in which the sum of the gain factors in Eq. (13) decreases as the initial tapping frequency increases. Even though the sensitivity to synchronization error, namely, the gain factor of $S_{E}$, decreases, the pair of subjects can synchronize their tapping motion due to the triangle configuration of the convergence region in Fig. 13.

\section{GENERAL DISCUSSION}

Engström et al. [5] studied the distribution of $S_{E}$ and its dependence on the signal frequency. They found a transition from reactive tapping to anticipatory tapping in the frequency region range of $0.5-1.0 \mathrm{~Hz}$. The transition region to the synchronization mode in the present mutual tapping experiments coincides with the frequency region at which Engström et al. found the transition to the anticipation mode. It would therefore support the idea that transitions to the anticipation mode with man-machine tapping and transitions to the synchronization mode in mutual tapping systems have the same kind of origin. The difference between the two experimental paradigms is that in mutual tapping, one has to anticipate the next tapping moment, which can be explained by the proportional control. The mode transition may be explained by the gradual shift of the gain factors in the proportional control based on $S_{E}$ and on $C_{E}$, i.e., the nonlinear nature of Eq. (14) showed that the gradual shift of gain factors gave rise to a discrete transition in $S_{E}$ as shown theoretically in Fig. 13 and experimentally in Fig. 5.

The synchronization models that have been proposed differ in their relative sensitivity to period correction and phase correction processes. For instance, Mates constructed a linear timing model based on error corrections, which aimed to explain the underlying mechanism for the synchronization process in mechanical tapping experiments $[15,16]$. However, he has to assume an internal time keeper to represent the cycle time. Note that in our modeling a rhythmic component was not assumed and a systematic model was presented in the combination model. In the tapping experiments to a rhythmic target, experimental verification of the control scheme was not clear since the synchronization error might be locked with respect to the rhythmic signal in the mechanical tapping experiments, whereas the mutual tapping experiments are symmetric by definition, and what seems fluctuation or noise in the mechanical tapping turns out to be a result of the feedback control based on the wide range of the synchronization errors (Fig. 7).

The interaction of two oscillators has been an interesting subject of nonlinear dynamical theory [17]. The neural network system, for example, can synchronize to generate a pulse overcoming inherent delay in each nerve cells. Recently, Ernst et al. analytically showed that the arbitrary small delays lead to stable in-phase behavior in the inhibitory coupling [13].

For the case of human behavior, there are dynamical system approaches that consider the action and the pacing sequence to be a system of weakly coupled oscillators in which the synchronization between the external stimuli and the human motion is explained by the coupling or entrainment of oscillators. Kelso observed an interesting phenomenon [18]. Under instructions to increase the frequency of out-ofphase, antisymmetrical motion, the subject's finger movements shifted abruptly to an in-phase symmetrical mode. Haken et al. proposed a double potential model to explain the mode transition of mutual finger motion from antiphase motion at lower frequencies to synchronized motion at higher frequencies [19]. Two oscillators coupled by the sinusoidal terms of the fundamental and higher harmonics can create a double minimal potential in the phase difference. In the present case, this model is not appropriate. The $S_{E}$ values at low frequencies in mutual tapping experiments correspond to a phase difference of $0.3 \mathrm{rad}$. To make a stable potential minimum at $0.3 \mathrm{rad}$, we must include a tenth harmonic in the coupling term, which appears to be impractical. Thus, in our study involving mutual tapping experiments, it is preferred to discuss the results based on explicit error correction.

Regarding the interpersonal synchronization, Nessler and Gilliland explained the phenomenon of synchronization during side-by-side walking in terms of unintentional entrainment, 
leaving further work to identify clearly the role of each mode of sensory information in the feedback loop that governs unintentional synchronization [10]. In the case of walking, the rhythmic component is inherent in the motion of a single person, and therefore the entrainment of the oscillator is the most probable explanation. In contrast to this phenomenon, the synchronization in mutual tapping is dependent on the feedback loop for the timing errors in going from tapping number $n$ to $n+1$. In addition, the mathematical model predicts synchronization without a rhythmic component.

In our previous work on mutual tracking experiments, a transition from the reactive mode to the synchronization mode was discovered [20]. The transition of the mode was located in the frequency region range of $0.5-1.0 \mathrm{~Hz}$. When tracking a continuously moving target along a circle, the mode transition was accompanied by a rhythmic component which was generated spontaneously in the subject pair. Even though the tracking behavior at low frequency was well described by feedback control of the positional error, the mathematical model which could predict the mode transition was left to a future study. In contrast, comprehensive understanding has been obtained to explain the mode transition in a simple feedback control law due to the discrete nature of the finger tapping in which only "timing" of the motion in the time dimension is involved. In the case of mutual tracking experiments in which "timing and spacing" of motion in the spatial and temporal dimensions are involved, the transition of the mode cannot be explained by only feedback control; feedforward control accompanied by the generation of a rhythmic component should be necessary to explain the synchronization in the higher-frequency region. Therefore, the nature of the anticipation in terms of the control law should be different, depending on the physical dimensions of the synchronization.

\section{CONCLUSION}

As the frequency in mutual tapping increases, we observed a mode transition from the alternative mode to the synchronization mode (Fig. 6). The tapping mechanism is found to be a proportional control of the cycle time correction based on the synchronization error (Fig. 7) and the cycle time error (Fig. 10). A mathematical model based on feedback control in the sensory-motor closed loop predicts a mode transition from the divergence to synchronization as the gain factors of the proportional control decease when the tapping frequency increases (Fig. 13). A key factor for the synchronization was the subtraction of the synchronization errors in the next tapping process. Note that no rhythmic component or internal representation is assumed in the model; the conditions of synchronization were shown to be a consequence of the coupled dynamics based on the subsequent feedback loop in the sensory-motor system.

\section{ACKNOWLEDGMENT}

This study was supported by the "Symbiotic Information Technology Research Project" at the Tokyo University of Agriculture and Technology.
[1] I. Miyake, Stud. Yale Psychol. Lab. 10, 1 (1902).

[2] W. Bair, R. J. Cavanaugh, A. M. Smith, and A. J. Movshon, J. Neurosci. 22, 3189 (2002).

[3] H. B. Repp, Psychonomic Bull. Rev. 12, 969 (2005).

[4] T. Radil, J. Ilmberger, and E. Pöppel, Experientia 46, 762 (1990).

[5] A. D. Engström, S. J. A. Kelso, and T. Holroyd, Hum. Mov. Sci. 15, 809 (1996).

[6] F. Ishida and Y. Sawada, Phys. Rev. Lett. 93, 168105 (2004).

[7] Y. Hayashi, Y. Tamura, K. Sase, K. Sugawara, and Y. Sawada, Trans. SICE 46, 391 (2010).

[8] H. U. Voss, Phys. Rev. E 61, 5115 (2000).

[9] N. Stepp, Exp. Brain Res. 198, 521 (2009).

[10] A. J. Nessler and S. Gilliland, Hum. Mov. Sci. 28, 772 (2009).

[11] A. J. Nessler and S. Gilliland, Gait Posture 31, 527 (2010).
[12] G. Rizzolatti and L. Craighero, Annu. Rev. Neurosci. 27, 169 (2004).

[13] U. Ernst, K. Pawelzik, and T. Geisel, Phys. Rev. Lett. 74, 1570 (1995).

[14] Y. Hayashi, K. Sugawara, and Y. Sawada, in Proceedings of the Fourth IMEKO TC 18 Symposium on Measurement, Analysis and Modeling of Human Functions (2010), p. 90 (unpublished).

[15] J. Mates, Biol. Cybern. 70, 463 (1994).

[16] J. Mates, Biol. Cybern. 70, 475 (1994).

[17] Y. Kuramoto, Chemical Oscillations, Waves, and Turbulence (Springer, Berlin, 1984).

[18] S. J. A. Kelso, Bull. Psychonomic Soc. 18, 63 (1981).

[19] H. Haken, S. J. A. Kelso, and H. Bunz, Biol. Cybern. 51, 347 (1985).

[20] Y. Hayashi and Y. Sawada, Phys. Rev. E (to be published). 